\title{
Can we have some more?
}

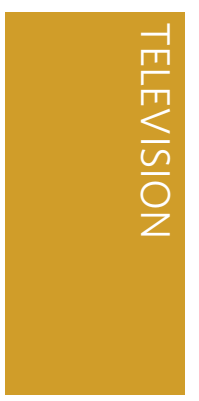

When such a dearth of material exists, the announcement that the $\mathrm{BBC}$ were to make a mainstream science series entirely focusing on chemistry was met with quite some enthusiasm in the community. However, once clear that Chemistry: A Volatile History would only be a three-episode run (at sixty minutes per programme; first shown 12 January 4 February 2010), my personal enthusiasm was tinged with dismay. How could even the most concise writing describe the volatile history of such an abiding discipline? (My personal history of volatile chemistry could easily fill double that time.) However, having watched this series on BBC Four (a digital-only service in the UK), I must first congratulate the producers for their choice of material to cover. Distilling 350 years of discovery, delusion, innovation and commercial success into 180 minutes is no mean feat.

Episode one begins with the founding of the new science of chemistry from the astrology-ridden roots of alchemy, and focuses on the work of Humphry Davy and his contemporaries in their pursuit of the idea of elements and their nature. With recreations of the chemical reactions used by these scientists, often making and using historical apparatus, the viewer is quickly thrown into a world of suspicious odours, life-threatening instability and academic rivalry. The introduction of phlogiston as the rationale for reactivity made for some uncomfortable viewing, especially as this delusion continued for so long, but the story was delivered with such enthusiasm that I couldn't look away. Much of the excitement is driven by the presenter; the infectiously animated Jim Al-Khalili (University of Surrey), whose willingness to endure everything from incandescent phosphorus to inhaling chlorine gas is to be commended. A certain measure of this comes from AlKhalili's evident personal curiosity.

Al-Khalili's background as a theoretical physicist actually supports the material presented in episode two, which focuses on the search for order amongst a seeming random array of elements. His examination of periodicity through a discussion of the properties of elements (including the odour of halogens and reactivity of alkali metals with water) is exceptionally well demonstrated, and helps the audience to decipher meaning in the periodic table. He then goes on to bring meaning to the numbers scattered around the table by dissecting the atom in a vivid and engaging manner, using simple visual aids to describe concepts such as valency. Most importantly, he conveys this information in a style enticing to both qualified chemists and the general public - certainly no mean feat.

The third and final instalment begins with an absorbing introduction to the industrialization of chemistry, focusing

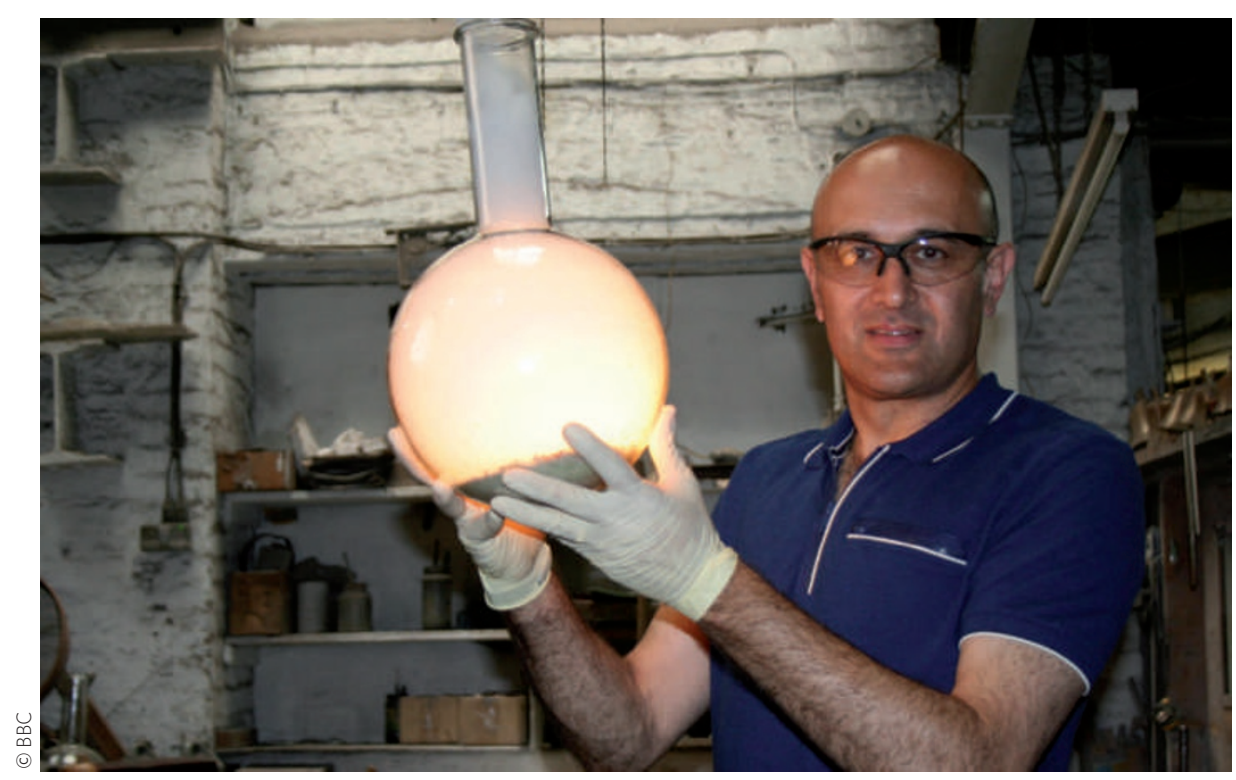

initially on the development of synthetic dyes. It's back to the lab for Al-Khalili, as he demonstrates the synthesis of Prussian blue, working with blood as a reagent. Examination of molecular structure follows this, with another excellent visual illustration - this time of isomerism, and why the connectivity of atoms is so important. In a reaction that I would have been terrified to re-enact, Al-Khalili performs an oxidation of diamond by heating the gems vigorously and throwing them into a Dewar of liquid oxygen - definitely what we'd describe as forcing conditions in the literature. The science is not only visceral, but truly beautiful, and leads into a brief discussion of carbon chemistry and its use in plastics.

However, most of the episode is dedicated to nuclear chemistry and the production of synthetic elements. After an absorbing introduction, this abrupt move into the somewhat esoteric area of neutron bombardment feels rather awkward. Perhaps it should come as no surprise, as this is AlKhalili's own research interest, but I think that this does a disservice to modern chemistry. Personally, I would have liked to see some mention of the development of modern pharmaceuticals (a thought that no doubt stems from my time in medicinal chemistry).

I must criticize the $\mathrm{BBC}$ for their choice of presenter - a practising chemist would surely have made a more natural choice (and there are many chemists with the demeanour and enthusiasm to present such a programme); the choice of a physicist also seems to shape the content of the series more to that discipline. Despite this, the final episode still makes for compelling popular science television, because Al-Khalili's passion for this subject is undeniable.

Concluding at this point leaves a slightly sour note in my mouth, but the series as a whole impresses. The vocation of the presenter aside, the production manages to entertain and inform on many levels, and proves that the $\mathrm{BBC}$ can make quality science documentaries without (the ever entertaining) Brian Cox - Britain's other telegenic physicist. However, with so few episodes, and so much more chemistry undiscussed, I'm left with one question can we have some more, please?

\section{REVIEWED BY PAUL DOCHERTY}

Paul Docherty is a chemist based in London, UK, and writes the blog TotallySynthetic.com 